\title{
ORIGINAL ARTICLE \\ Gut-derived lipopolysaccharide augments adipose OPEN ACCESS macrophage accumulation but is not essential for impaired glucose or insulin tolerance in mice
}

\author{
Robert Caesar, ${ }^{1,2}$ Christopher S Reigstad, ${ }^{1,2}$ Helene Kling Bäckhed, ${ }^{1,2}$ \\ Christoph Reinhardt, ${ }^{1,2}$ Maria Ketonen, ${ }^{1,2}$ Gunnel Östergren Lundén, ${ }^{1,2}$ \\ Patrice D Cani, ${ }^{3}$ Fredrik Bäckhed ${ }^{1,2}$
}

\begin{abstract}
- Additional materials are published online only. To view these files please visit the journal online (http://dx.doi.org/ 10.1136/gutjnl-2011-301689).

${ }^{1}$ Sahlgrenska Center for Cardiovascular and Metabolic Research/Wallenberg Laboratory, Gothenburg, Sweden

${ }^{2}$ Department of Molecular and Clinical Medicine, University of Gothenburg, Gothenburg, Sweden

${ }^{3}$ Université catholique de Louvain, Louvain Drug Research Institute, Metabolism and Nutrition Research Group, Brussels, Belgium
\end{abstract}

\section{Correspondence to} Dr Fredrik Bäckhed, Wallenberg Laboratory, Sahlgrenska University Hospital, S-413 45 Gothenburg, Sweden; fredrik.backhed@wlab.gu.se

Revised 27 February 2012 Accepted 12 March 2012

Published Online First 25 April 2012

\section{ABSTRACT \\ Background Obesity is associated with accumulation of macrophages in white adipose tissue (WAT), which contribute to the development of insulin resistance. Germ-free (GF) mice have reduced adiposity and are protected against diet-induced obesity, \\ Objective To investigate whether the gut microbiota and, specifically, gut-derived lipopolysaccharide (LPS) promote WAT inflammation and contribute to impaired glucose metabolism. \\ Method Macrophage composition and expression of proinflammatory and anti-inflammatory markers were compared in WAT of GF, conventionally raised and Escherichia coli-monocolonised mice. Additionally, glucose and insulin tolerance in these mice was determined.}

Results The presence of a gut microbiota resulted in impaired glucose metabolism and increased macrophage accumulation and polarisation towards the proinflammatory M1 phenotype in WAT Monocolonisation of GF mice for 4 weeks with E.coli W3110 or the isogenic strain MLK1067 (which expresses LPS with reduced immunogenicity) resulted in impaired glucose and insulin tolerance and promoted M1 polarisation of CD11b cells in WAT. However, colonisation with E.coli W3110 but not MLK1067 promoted macrophage accumulation and upregulation of proinflammatory and anti-inflammatory gene expression as well as JNK phosphorylation.

Conclusion Gut microbiota induced LPS-dependent macrophage accumulation in WAT, whereas impairment of systemic glucose metabolism was not dependent on LPS. These results indicate that macrophage accumulation in WAT does not always correlate with impaired glucose metabolism

\section{INTRODUCTION}

Obesity is associated with insulin resistance and chronic low-grade inflammation characterised by accumulation of proinflammatory M1 macrophages in white adipose tissue (WAT) ${ }^{1-3}$ Recent evidence showing that germ-free (GF) mice are protected against diet-induced obesity and exhibit reduced WAT inflammation and insulin resistance ${ }^{4-6}$ indicates a role for gut microbiota in the link between obesity, inflammation and insulin resistance. Furthermore, obese humans have reduced gut microbial diversity, ${ }^{7}$ and modulation of

\section{Significance of this study}

What is already known on this subject?

- The gut microbiota is altered in obese mice and humans.

- Germ-free mice have reduced adiposity and are resistant to diet-induced obesity.

- Obesity and insulin resistance are associated with inflamed adipose tissue.

What are the new findings?

- The gut microbiota promotes adipose inflammation.

- Macrophage recruitment to adipose tissue requires lipopolysaccharide (LPS).

- E.coli promotes macrophage polarisation to a proinflammatory (M1)-biased phenotype, which is independent of LPS.

- E.coli-induced impairment of glucose and insulin tolerance in mice does not require proinflammatory LPS.

How might it impact on clinical practice in the foreseeable future?

- Understanding the mechanisms by which the gut microbiota induces metabolic diseases may provide new diagnostic and therapeutic targets to treat these life-threatening diseases. It also demonstrates the power of combining bacterial genetics with gnotobiotic animal models to study host metabolism.

the gut microbiota either by antibiotics or prebiotics improves metabolic and inflammatory properties, suggesting a direct role of the gut microbiota in metabolic diseases. ${ }^{8-10}$

It is not clear which microbial factors promote WAT inflammation but the bacterial endotoxin lipopolysaccharide (LPS) has been suggested as a key factor. Obese mice and humans with type 2 diabetes have increased plasma LPS concentrations $^{11}{ }^{12}$ and LPS promotes secretion of proinflammatory cytokines by activating Toll-like receptor (TLR) $4 .^{13}$ Furthermore, chronic subcutaneous infusion with LPS results in increased macrophage accumulation and expression of inflammatory markers in WAT and insulin 
resistance in the liver. ${ }^{11}$ LPS therefore constitutes a putative link between microbially induced inflammation, obesity and metabolic diseases. Here we use gnotobiotic mice coupled with bacterial genetics to investigate if LPS originating from E.coli in the gut is sufficient to promote glucose and insulin tolerance and macrophage accumulation in WAT.

\section{MATERIAL AND METHODS}

\section{Mice and bacterial strains for monocolonisation}

GF male Swiss Webster mice, 10-12 weeks old, were maintained in flexible film isolators under a strict $12 \mathrm{~h}$ light cycle. GF status was verified regularly by anaerobic culturing in addition to PCR for bacterial $16 S$ rDNA. ${ }^{14}$ Both GF and conventionally raised (CONV-R) mice were fed autoclaved chow diet (Labdiet) ad libitum.

E.coli strains W3110 and MLK1067 (W3110 waaN) were used for monocolonisation. Both strains express type-1 fimbriae but not P-pili or $\alpha$-haemolysin. ${ }^{15}$ W3110 is a well-characterised wild-type strain and the isogenic strain MLK1067 was chosen because it has reduced immunogenicity compared with W3110. ${ }^{15-17}$ GF mice were inoculated for 4 weeks with E.coli W3110 or MLK1067 $7^{18}$ $\left(3-4 \times 10^{9}\right.$ colony-forming units (CFU) per cage; $\sim 10^{9}$ per mouse). Final colonisation densities were determined from mouse ceca by serial dilution and plating on Luria-Bertani agar.

Epididymal WAT and plasma were harvested after a $5 \mathrm{~h}$ fast.

\section{DEXA, insulin and glucose tolerance tests and measurements of plasma leptin, lipids and insulin levels}

DEXA was performed as earlier described. ${ }^{19}$ Insulin and glucose tolerance tests were performed by injecting insulin $(0.75 \mathrm{U} / \mathrm{kg}$ body weight) or glucose $(2 \mathrm{~g} / \mathrm{kg}$ body weight), respectively, intraperitoneally after a $5 \mathrm{~h}$ fast. Tail blood samples were collected at 0, 30, 60, 90 and $120 \mathrm{~min}$ and blood glucose levels were determined using a HemoCue glucose 201+ analyser (HemoCue, Ängelholm, Sweden). Fasting leptin, free fatty acids, triglycerides and insulin were measured with kits from Meso Scale (Gaithersburg, Maryland, USA), Abcam (Cambridge, Massachusetts, USA), Thermo Scientific (Waltham, Massachusetts, USA) and Crystal Chem (Downers Grove, Illinois, USA), respectively, according to the manufacturers' protocols.

\section{Immunohistochemistry of WAT}

Paraffin-embedded epididymal WAT sections $(5 \mu \mathrm{m})$ were deparaffinised and processed for antigen retrieval with a 2100 Retriever using $1 \times$ DIVA solution and Hot Rinse (HistoLab Products AB, Gothenburg, Sweden). Endogenous peroxidase activity was quenched by incubating slides in $0.3 \% \mathrm{H}_{2} \mathrm{O}_{2}$ in phosphate-buffered saline (PBS) for 30 min before blocking in $5 \%$ rabbit serum, $1 \%$ bovine serum albumin and $0.1 \%$ Triton $\mathrm{X}$ 100 at room temperature for $30 \mathrm{~min}$. Macrophages were stained with MAC-2/galectin-3 antibody (online supplementary table S1) diluted 1:500 in blocking buffer overnight at $4^{\circ} \mathrm{C}$, which was detected with a biotinylated anti-rat $(10 \mu \mathrm{g} / \mathrm{ml})$ antibody. Immune complexes were detected by VECTASTAIN Elite ABC reagent followed by colour development using NovaRed substrate solution (Vector Laboratories, Burlingame, California, USA), according to the manufacturer's instructions, and counterstained with haematoxylin. Crown-like structures were counted in 15-40 $\mathrm{mm}^{2}$ of histological sections per mouse.

\section{Culture and stimulation of RAW 264.7 macrophages}

RAW 264.7 macrophages (ATCC, Manassas, Virginia, USA) were maintained in Dulbecco's modified Eagle's medium (PAA Laboratories, Pasching, Austria) containing $100 \mathrm{U} / \mathrm{ml}$ penicillin,
$100 \mathrm{mg} / \mathrm{ml}$ streptomycin, $2 \mathrm{mM}$ L-glutamine, $2 \mathrm{mM}$ sodium pyruvate and $10 \%$ fetal calf serum. Cells were seeded at equal densities and upon reaching confluence they were washed and incubated with heat-killed E.coli or control medium. Tumour necrosis factor $\alpha$ was measured in the supernatant by Meso Scale immunoassay after $4 \mathrm{~h}$ incubation according to the manufacturer's protocol. In separate experiments, cells were lysed in RTL buffer (Qiagen, Hilden, Germany) and snap frozen in liquid nitrogen.

\section{Isolation of adipose tissue macrophages}

Epididymal WAT was minced thoroughly and resuspended in $15 \mathrm{ml}$ digestion solution (7 $\mathrm{ml}$ Hanks' solution, $3 \mathrm{ml} 7.5 \%$ bovine serum albumin (BSA), and $20 \mathrm{mg}$ collagenase type II (Sigma Aldrich, St Louis, Missouri, USA)). Tissue digestion was performed at $37^{\circ} \mathrm{C}$ using a shaker at $120 \mathrm{rpm}$ for $40 \mathrm{~min}$. Adipocytes were removed and the stroma vascular fraction was centrifuged $\left(1200 \mathrm{rpm}, 4^{\circ} \mathrm{C}, 5 \mathrm{~min}\right)$ and subsequently resuspended in $1 \mathrm{ml}$ selection buffer (PBS, $2 \mathrm{mM}$ EDTA, and $0.5 \%$ BSA). CD11b cells were selected using CD11b microbeads (Miltenyi Biotec, Auburn, California, USA), according to the manufacturer's instructions.

\section{Flow cytometry analysis}

Adipose tissue macrophages were incubated with BD Fc Block (BD Biosciences, Franklin Lakes, New Jersey, USA) for 5 min, followed by incubation with monoclonal antibodies (online supplementary table S1). After washing, cells were analysed using an Accuri C6 flow cytometer with CFlow Plus analysis software (Accuri Cytometers, Ann Arbor, Michigan, USA). Nonspecific antibodies conjugated to the corresponding fluorochromes were used as negative controls, and the fluorescence threshold was set so that $2.5 \%$ of the total cells were positive for fluorescence. The percentage of positive cells for the antibodies of interest was determined by the percentage of cells exceeding the threshold obtained with non-specific antibodies.

\section{LPS analysis}

Blood was collected from the mouse portal vein using a pyrogenfree syringe/needle, and plasma was immediately isolated and frozen in liquid nitrogen. LPS concentration was measured using Endosafe-MCS (Charles River, Lyon, France) based on the limulus amoebocyte lysate (LAL) kinetic chromogenic methodology that measures colour intensity directly related to the endotoxin concentration in a sample. Plasma was diluted $1 / 10$ with endotoxin-free buffer to minimise interferences in the reaction (inhibition or enhancement) and heated for $15 \mathrm{~min}$ at $70^{\circ} \mathrm{C}$. Each sample was diluted with endotoxin-free LAL reagent water (Charles River) and treated in duplicate and two spikes for each sample were included in the determination. ${ }^{20}$

To determine the LPS content in the diet, randomly selected pellets were ground in endotoxin-free tubes (Greiner, Frickenhausen, Germany). The ground diet $(100 \mathrm{mg}$ ) was sonicated in $10 \mathrm{ml}$ endotoxin-free LAL reagent water (Charles River) and filtered $(0.22 \mu \mathrm{m}$, Millipore). The solution was diluted in endotoxin-free LAL reagent water and LPS concentration was measured using Endosafe-MCS. Each sample was treated in duplicate and two spikes for each sample were included in the determination.

The lower limit of detection was 0.005 endotoxin units $(E U) / \mathrm{ml}$.

\section{Quantitative RT-PCR}

RNA was isolated using RNeasy kit with on-column DNase treatment (Qiagen, Hilden, Germany). cDNA templates were 
synthesised from total RNAs using the high-capacity cDNA reverse transcription kit (Applied Biosystems, Foster City, California, USA) according to the manufacturer's instructions. qRTPCR assays were performed in $25 \mu$ reactions containing $1 \times$ SYBR Green Master Mix buffer (Thermo Scientific, Waltham, Massachusetts, USA), and $900 \mathrm{nM}$ gene-specific primers (300 nM primer concentrations were used to assess L32 transcripts). Gene expression data were normalised to the ribosomal protein L32. Primer sequences are reported in online supplementary table S2.

\section{Immunoblot analysis}

Protein extracts $(20 \mu \mathrm{g})$ were loaded onto 10\% NuPAGE Bis-Tris gels (Invitrogen, Carlsbad, California, USA) and blotted to Hybond P PVDF membranes (Invitrogen). After $1 \mathrm{~h}$ blocking in PBS Tween-20 (PBST) supplemented with 5\% fat-free milk powder the membranes were incubated overnight with primary antibodies diluted 1:1000 in blocking buffer and subsequently washed in PBST, incubated for $1 \mathrm{~h}$ with secondary antibodies diluted 1:2000 in blocking buffer, and finally washed in PBST (antibodies are listed in table S1). Proteins were detected with LumiGLO blotting detection kit (Cell Signaling, Danvers, Massachusetts, USA).

\section{Statistical analysis}

Data are represented as mean \pm SEM. Analyses between groups of two were determined by Student $t$ test, whereas comparisons of three or more groups were analysed by one-way analysis of variance with ad hoc Bonferroni post tests using GraphPad Prism 5 software.

RESULTS AND DISCUSSION

Gut microbiota increases adiposity, impairs glucose and insulin tolerance and increases macrophage accumulation in WAT, even in the absence of a high-fat diet

It has previously been shown that colonisation of C57BL/6 mice results in a significant increase in adiposity and impaired glucose and insulin tolerance even in the absence of a high-fat diet. ${ }^{19}$ Here we used Swiss Webster mice as they are generally bigger and have increased adiposity compared with C57BL/6 mice. As expected, we showed significant increases in epididymal WAT and body fat in CONV-R compared with GF mice on a chow diet at 12 weeks of age (figure 1A,B). Adiposity in the CONV-R mice was similar to that previously reported for $\mathrm{C} 57 \mathrm{BL} / 6$ mice fed a high-fat diet for 8 weeks $(33 \pm 2$ vs $37 \pm 5 \mathrm{mg}$ epididymal WAT/g body weight, respectively), ${ }^{4}$ confirming Swiss Webster mice as a suitable model for mild obesity. Furthermore, by using a chow diet, we avoided confounding factors such as saturated fatty acids present in high-fat diets. Colonisation of 12 -week-old GF mice with a normal microbiota for 14 days increased body fat to the level seen in CONV-R mice (figure 1B). As expected, we observed increased plasma leptin and insulin levels as well as impaired glucose and insulin tolerance in 12-14-week-old CONV-R mice compared with age-matched GF mice on a chow diet (figure $1 \mathrm{C}-\mathrm{F}$ ). No differences in plasma levels of free fatty acids or triglycerides were seen (data not shown).
A

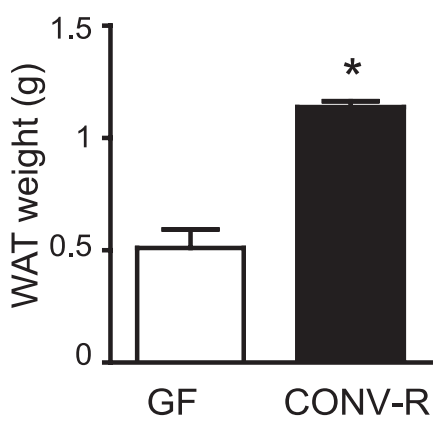

B

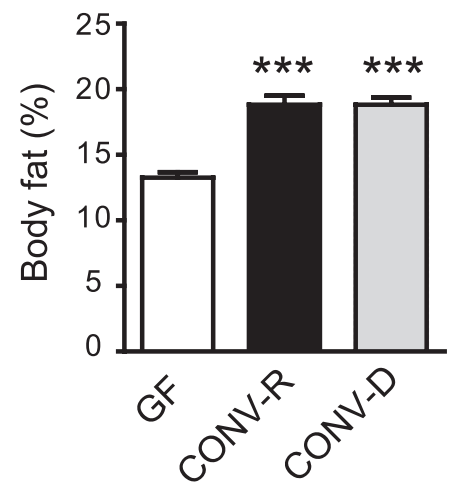

C

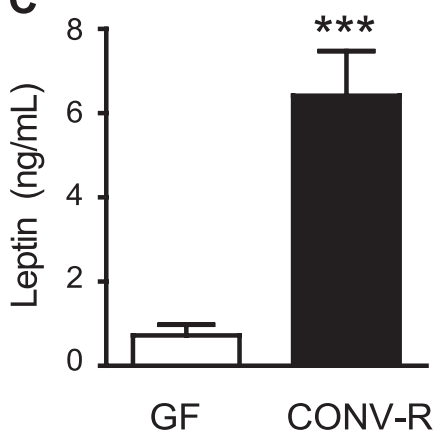

D

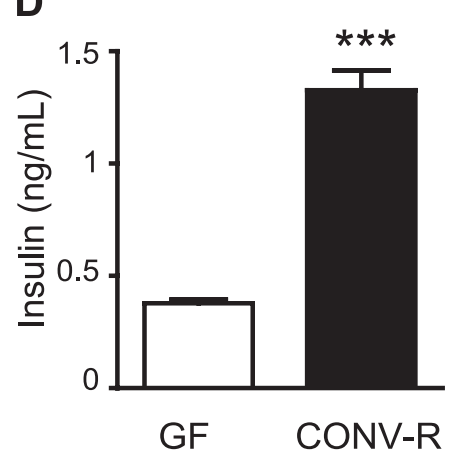

E

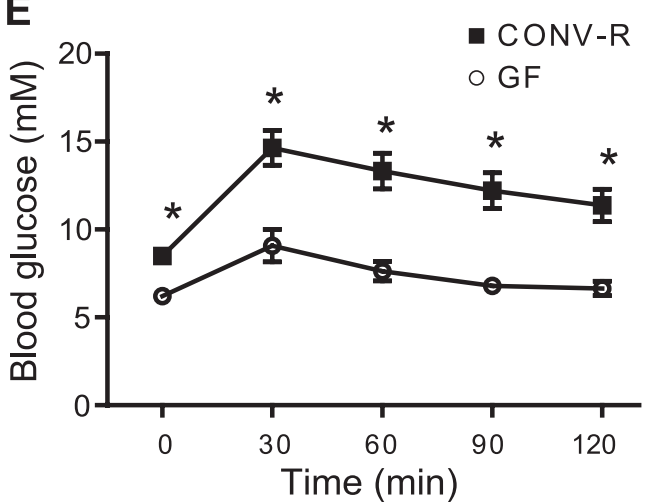

$\mathbf{F}$

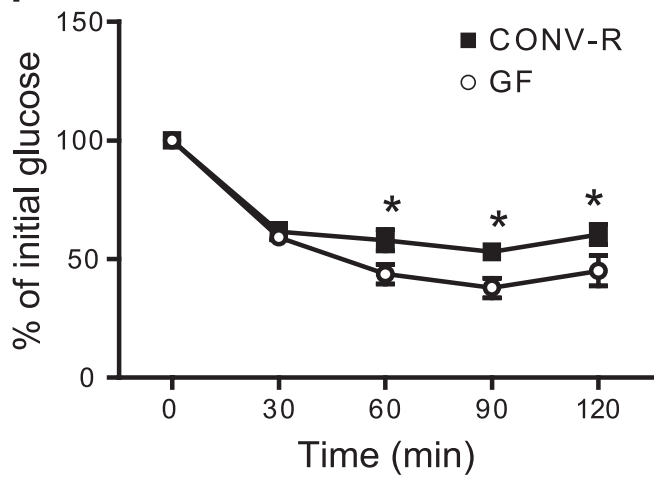

Figure 1 Gut microbiota increases adipose tissue weight and impairs glucose homoeostasis. (A) Epididymal white adipose tissue (WAT) weight in germ-free (GF) and conventionally raised (CONV-R) mice ( $n=8-9$ mice per group). (B) Body fat determined by DEXA in GF and CONV-R mice and in GF mice colonised with a normal microbiota for 14 days (CONV-D) ( $n=8-10$ mice per group). (C) Fasting plasma leptin and (D) insulin levels in GF and CONV-R mice ( $n=7-8$ mice per group). (E) Glucose $(n=8-9$ mice per group) and (F) insulin tolerance in GF and CONV-R mice ( $n=5-6$ mice per group). Mean values \pm SEM are plotted; ${ }^{*} p<0.05,{ }^{* *} p<0.001$ versus GF. 
To investigate the effect of gut microbiota on macrophage accumulation in WAT, we stained epididymal WAT for MAC-2 and showed increased formation of crown-like structures (CLS) in CONV-R mice compared with GF mice (figure 2A,B). CLS are histological elements representing accumulation of macrophages around dead adipocytes, ${ }^{21}$ and abundance of CLS has been shown to correlate with metabolic dysfunction in obese individuals. $^{22}$ qRT-PCR analysis of WAT showed increased expression of the pan-macrophage marker Emr1 mRNA (which encodes EGF-like module receptor, also known as F4/80) in CONV-R mice compared with GF mice (figure 2C). FACS analysis of CD11b adipose tissue macrophages with antibodies against CD11c (M1) and CD209 (M2) showed an increased M1/M2 ratio in WAT from CONV-R mice compared with GF mice (figure 2D, online supplementary figure S1A,B) and an increased number of CD11c cells/g WAT (figure 2E). There was also a trend towards an increased number of CD209 cells/g WAT (figure $2 \mathrm{E}, \mathrm{p}=0.06$ ). $\mathrm{qRT}-\mathrm{PCR}$ analysis showed that both the proinflammatory cytokines Tnf $\alpha$ and Saa3 (figure 2F,G) and the anti-inflammatory markers $\mathrm{Mgll}$ and $\mathrm{Il}-10$ (figure $2 \mathrm{H}, \mathrm{I}$ ) were increased in WAT from CONV-R mice compared with GF mice. These data suggest that the increased ratio of $M 1$ macrophages is caused primarily by increased infiltration of these cells.

\section{Gut microbiota increases plasma levels of LPS}

Importantly, as we used a chow diet, the differences in glucose and insulin tolerance as well as macrophage accumulation and inflammation in WAT between CONV-R and GF mice could not be attributed to saturated fatty acids in the diet. We therefore hypothesised that these differences might be caused by components of the microbiota such as LPS. Indeed, we showed raised LPS levels (ie, metabolic endotoxaemia) in the plasma of CONV-R mice compared with GF mice on a chow diet (figure 2J). The diet contained $1.5 \mathrm{EU} \mathrm{LPS} / \mathrm{mg}$, which could explain the low levels of LPS present in the plasma of GF mice.
A
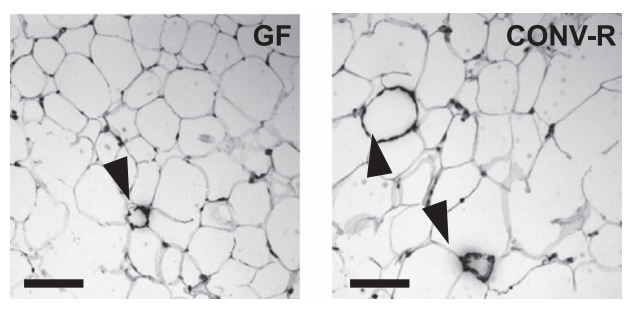

D

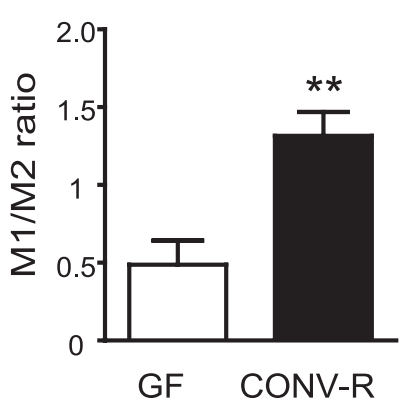

G

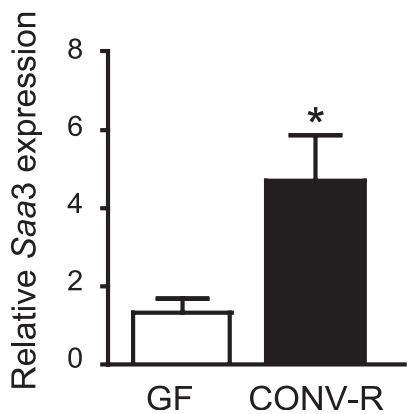

E

H
B
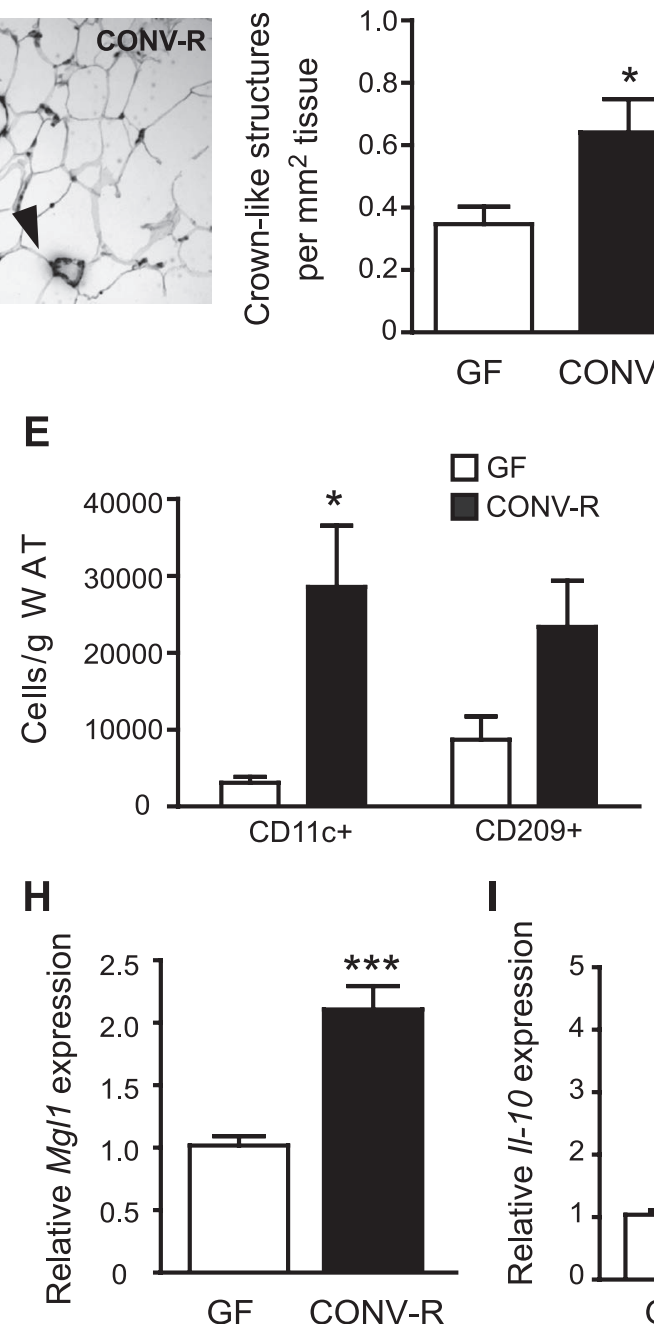

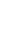

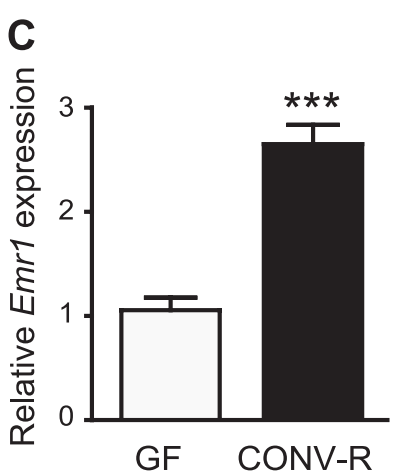

F

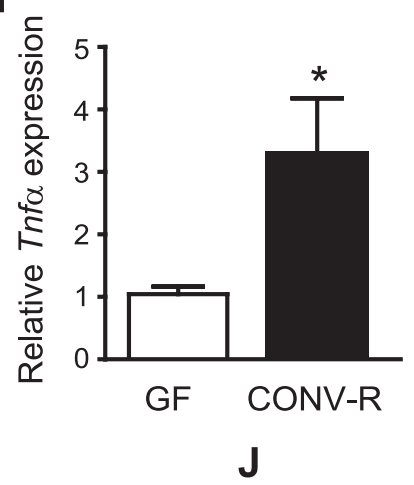

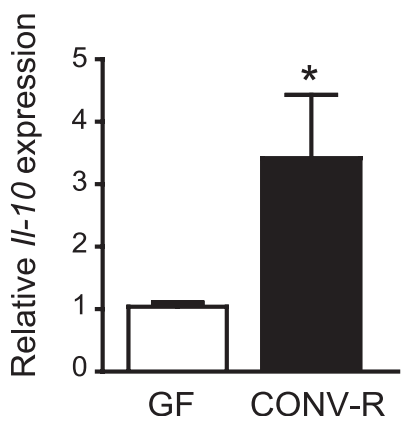

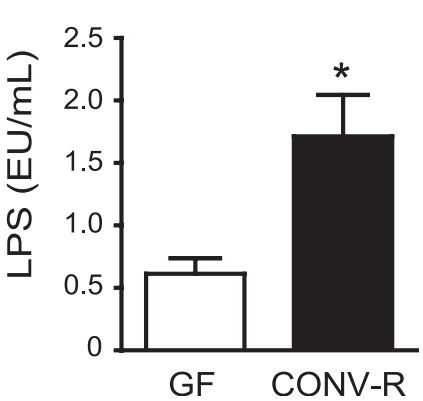

Figure 2 Gut microbiota increases crown-like structure (CLS) formation, M1/M2 ratio and expression of proinflammatory and anti-inflammatory cytokines in white adipose tissue (WAT). (A) Representative MAC-2 immunostaining of WAT from germ-free (GF) and conventionally raised (CONV-R) mice. Arrowheads indicate CLS. Scale bars $=100 \mu \mathrm{m}$. (B) Quantification of CLS ( $\mathrm{n}=9$ mice per group). (C) qRT-PCR analysis of Emr1 expression in WAT from GF and CONV-R mice ( $n=8-9$ mice per group). (D) Ratio between WAT M1 and M2 macrophages determined by flow cytometry with antibodies against CD11c conjugated with phycoerythrin and CD209 conjugated antigen presenting cells, respectively ( $\mathrm{n}=4$ (GF) and 8 (CONV-R)). (E) Number of CD11c (M1) and CD209 (M2) macrophages per gram WAT ( $\mathrm{n}=4)$. (F-l) qRT-PCR analysis of Tnf $\alpha$, Saa3, Mgl1 and II-10 expression in WAT from GF and CONV-R mice ( $n=8-9$ mice per group). (J) Lipopolysaccharide (LPS) levels in blood sampled from the portal vein of GF ( $n=9$ ) and CONV-R $(n=15)$ mice. Mean values \pm SEM are plotted; ${ }^{*} p<0.05,{ }^{* *} p<0.01,{ }^{* *} p<0.001$ versus GF. 
Colonisation with E.coli promotes adiposity and glucose and insulin tolerance independently of LPS

LPS has been associated with obesity and type 2 diabetes in mice and humans ${ }^{9} 12$ and subcutaneous administration of LPS promotes adiposity and a slight impairment of glucose metabolism. ${ }^{11}$ However, little is known about how LPS from the gut affects metabolism. We therefore investigated the importance of gut-derived LPS in mediating glucose and insulin tolerance and macrophage accumulation and inflammation by colonising GF mice with either E.coli W3110 or the isogenic mutant MLK1067, a strain with reduced immunogenicity owing to its penta-acylated lipid A component, ${ }^{15}$ for 4 weeks. We first confirmed that the mutant exhibited reduced capacity to induce Tnf $\alpha$ expression and tumour necrosis factor $\alpha$ secretion in murine RAW 264.7 macrophages (online supplementary figure S2A,B), and showed that W3110 and MLK1067 colonised the mouse gut approximately to the same bacterial density $\left(1.1 \times 10^{10}\right.$ and $1.5 \times 10^{10}$ CFU/ml for W3110 and MLK1067, respectively).

As expected, colonisation with E.coli W3110 but not MLK1067 increased plasma LPS levels compared with GF mice (figure 3A). However, colonisation with either E.coli strain increased epididymal WAT weight compared with GF mice (figure 3B). Plasma levels of leptin and insulin were higher in mice colonised with either strain compared with GF mice, with the highest levels found in mice colonised with MLK1067 (figure 3C,D). No differences in plasma levels of free fatty acids or triglycerides were observed with colonisation (data not shown) and no difference in food intake was seen (data not shown). Colonisation with either strain resulted in impaired glucose and insulin tolerance (figure $3 \mathrm{E}, \mathrm{F}$ ).
These findings show that monocolonisation with E.coli, even in the absence of wild-type LPS, is sufficient to increase adiposity and impair insulin and glucose tolerance. Other microbial components, such as peptidoglycan, may be important factors in modulating host glucose metabolism and adiposity. For example, mice deficient in the peptidoglycan receptors Nod1 and Nodz are protected from high-fat dietinduced inflammation and insulin intolerance, and activation of NOD1 causes insulin resistance. ${ }^{23}$

\section{Gut-derived LPS increases macrophage accumulation and proinflammatory and anti-inflammatory markers in WAT}

Colonisation with E.coli W3110, but not MLK1067, resulted in increased CLS formation (figure 4A,B) and increased expression of Emr1 (F4/80) (figure 4C). The observation that LPS is required for macrophage accumulation but not for adiposity is in agreement with earlier studies in which mice lacking CD14 or with a haematopoietic cell-specific TLR4 deletion (and hence reduced LPS signalling) develop obesity on a high-fat diet but show markedly reduced adipose tissue macrophage content. ${ }^{24}$

To examine whether the increased macrophage infiltration in WAT from mice colonised with W3110 was associated with increased inflammation, we analysed the M1/M2 ratio and levels of proinflammatory and anti-inflammatory cytokines in WAT. The M1/M2 ratio increased approximately twofold after colonisation with either E.coli strain, but the increase was only significant in mice colonised with W3110 (figure 4D, online supplementary figure S3A,B). The number of both CD11c and CD209 cells was increased in mice colonised with W3110 (figure 4E) and accordingly, we also observed increased
A

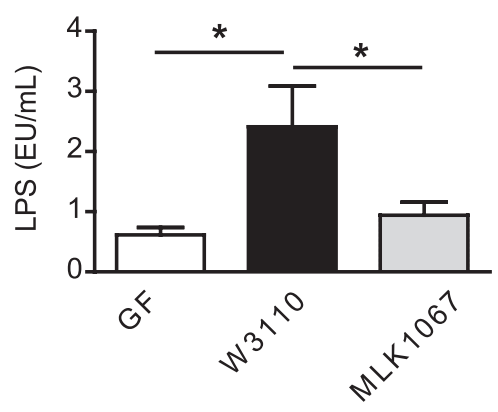

D

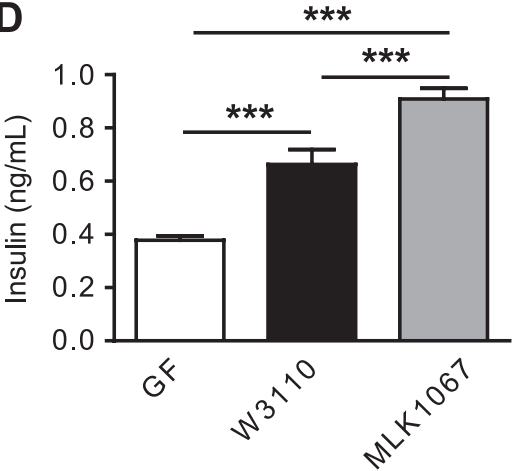

B

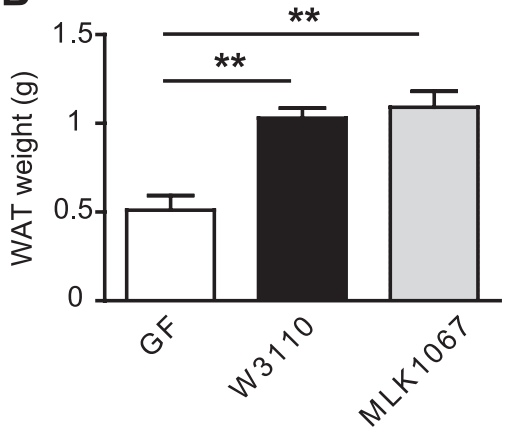

E

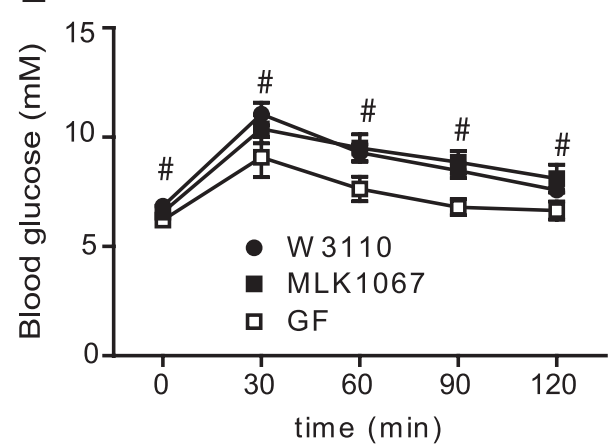

C

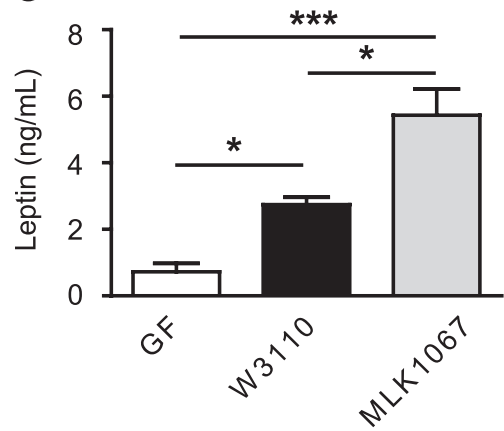

$\mathbf{F}$

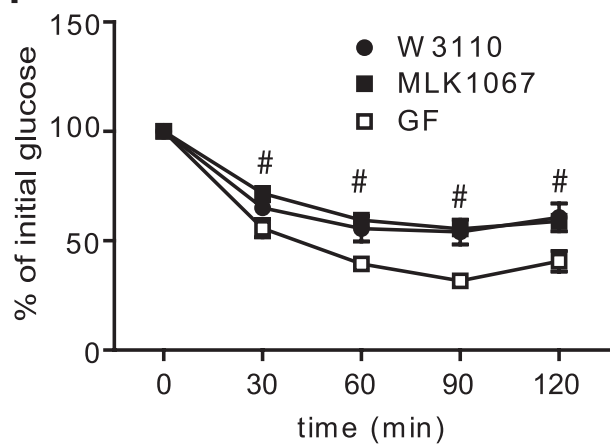

Figure 3 E.coli colonisation of germ-free (GF) mice increases white adipose tissue (WAT) weight and impairs glucose and insulin tolerance. (A) Lipopolysaccharide (LPS) levels in blood sampled from the portal vein of GF mice and mice colonised for 4 weeks with W3110 or MLK1067 ( $n=5-6$ mice per group). (B) Epididymal WAT weight ( $n=9-10$ mice per group). (C) Fasting plasma leptin and (D) insulin levels in GF and conventionally raised (CONV-R) mice ( $n=9-10$ mice per group). (E) Glucose tolerance test $(n=9-10$ mice per group). (F) Insulin tolerance test ( $n=5-6$ mice per group). Mean values \pm SEM are plotted; ${ }^{*} \mathrm{p}<0.05 ;{ }^{* *} \mathrm{p}<0.01 ;{ }^{* * *} \mathrm{p}<0.001$ versus GF in $\mathrm{A}-\mathrm{D} ; \# \mathrm{p}<0.05$ vs GF for both W3110 and MLK1067 in panels $\mathrm{E}$ and $\mathrm{F}$. 
A
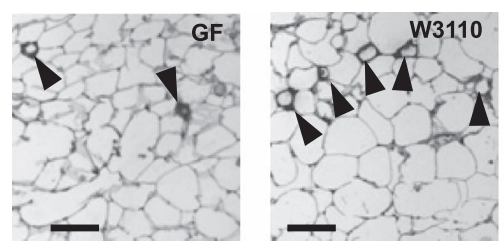

B

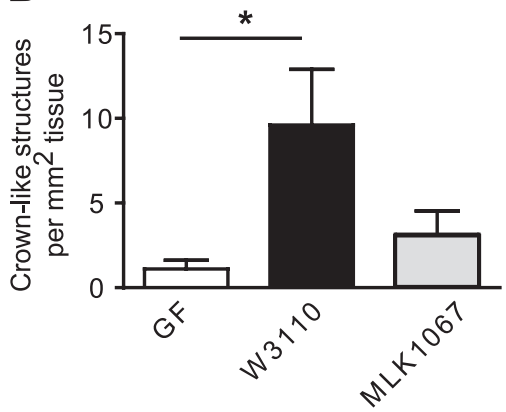

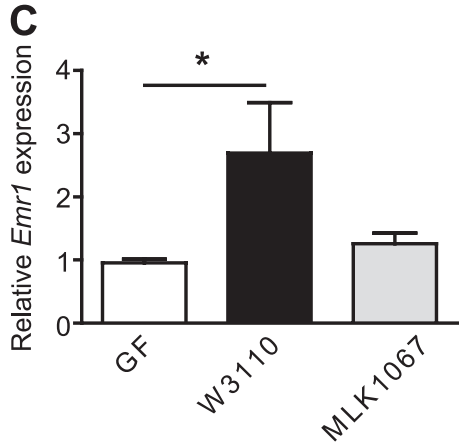

F

F

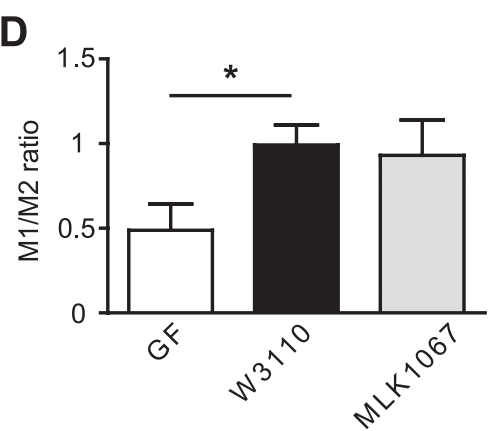

G

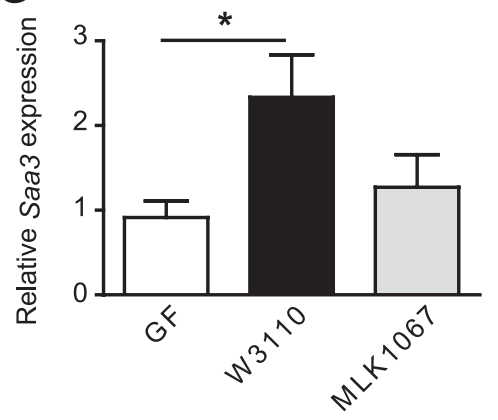

J

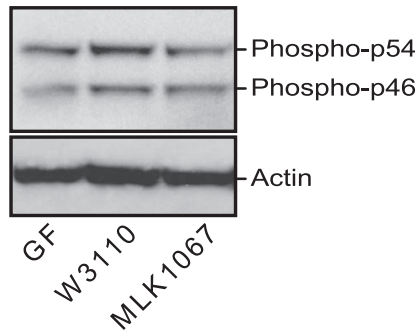

E

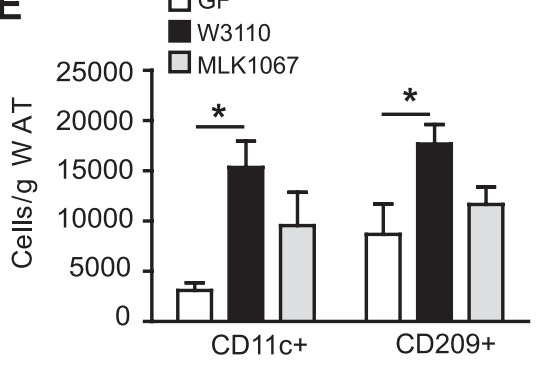

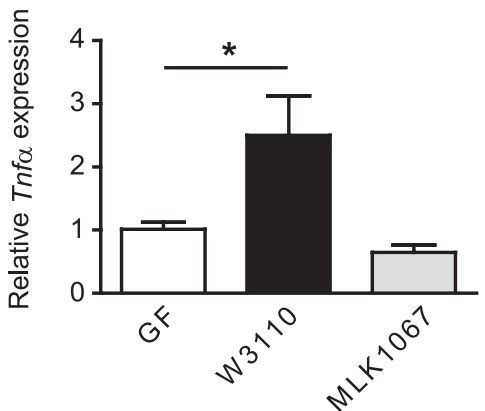

I

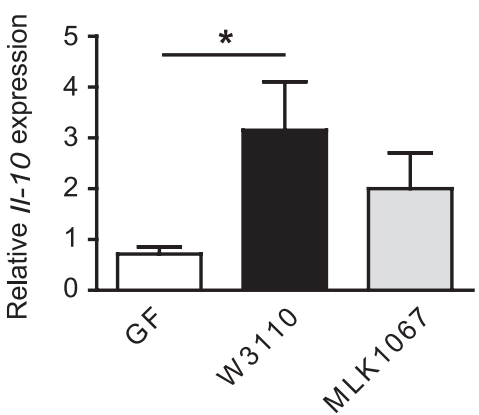

H

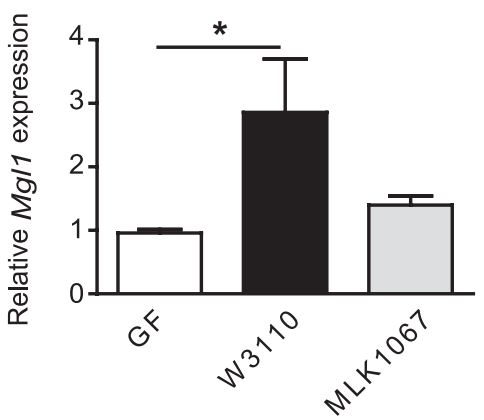

K

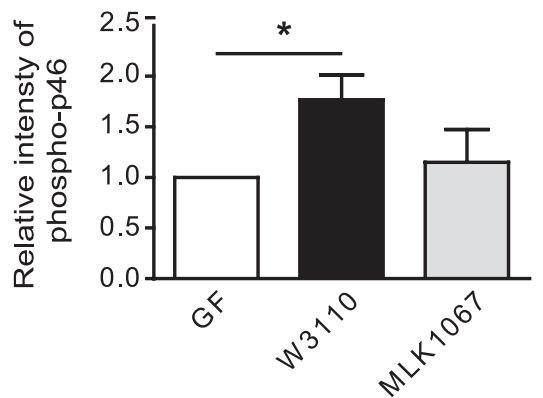

Figure 4 Gut-derived lipopolysaccharide (LPS) increases crown-like structure (CLS) formation, M1/M2 ratio and expression of proinflammatory and anti-inflammatory cytokines in white adipose tissue (WAT). (A) Representative immunostaining of MAC-2 in WAT from germ-free (GF) mice and mice colonised for 4 weeks with W3110 or MLK1067. Arrowheads indicate CLS. Scale bars $=100 \mu \mathrm{m}$. (B) Quantification of CLS ( $\mathrm{n}=4-5 \mathrm{mice}$ per group). (C) qRT-PCR analysis of Emr1 (F4/80) in WAT ( $\mathrm{n}=6-7$ mice per group). (D) Ratio between M1 and M2 activated macrophages in WAT determined by flow cytometry with antibodies against CD11c and CD209 conjugated (n=4 (GF) and 9 (W3110 and MLK1067)). (E) Number of CD11c ${ }^{+}$(M1) and CD209 (M2) macrophages per gram WAT ( $n=4-5)$. (F-I) qRT-PCR analysis of Tnf $\alpha$, Saa3, Mgl1 and II-10 expression in WAT ( $\mathrm{n}=5-6$ mice per group). (J) Total protein extracts from WAT were analysed by immunoblotting with antibodies against phospho-JNK and actin. (K) Relative quantification of the phospho-p46 form of JNK normalised to actin ( $n=6$ mice per group). Mean values \pm SEM are plotted; ${ }^{*} p<0.05$.

expression of both the proinflammatory markers $\operatorname{Tnf} \alpha$ and Saa3 and the anti-inflammatory markers $\mathrm{Mgll}$ and $\mathrm{Il}-10$ in WAT from W3110 colonised mice (figure 4F-I). Furthermore, immunoblot analysis revealed elevated levels of phosphorylated JNK (figure $4 \mathrm{~J}, \mathrm{~K})$ in WAT from W3110 colonised mice, indicative of increased inflammatory signalling. In contrast, none of these markers were increased by MLK1067 (figure 4F-K). The observation that an increased $M 1 / M 2$ ratio is not necessarily reflected in the balance between proinflammatory and anti-inflammatory cytokines demonstrates the complex nature of macrophage polarisation and shows that multiple markers are required to study the inflammatory status of WAT. 


\section{CONCLUSION}

Taken together, the gut microbiota affects host metabolism by several mechanisms that, in combination, may contribute to obesity and have profound effects on glucose metabolism. Here we demonstrate that LPS is sufficient to promote macrophage infiltration but is not essential for the impaired glucose metabolism associated with gut colonisation. Other microbial components have previously been shown to affect glucose and insulin tolerance, ${ }^{23} 25$ thus uncoupling LPS and WAT inflammation from impaired glucose metabolism. However, it is important to note that TLR4 recognition of LPS differs between mice and humans: penta-acylated MLK1067 LPS functions as a weak agonist for mouse TLR4 but is a potent antagonist for human TLR4. ${ }^{17} 26$

Acknowledgements We thank Carina Arvidsson and Anna Hallén for superb technical assistance and Rosie Perkins (Wallenberg Laboratory at University of Gothenburg) for editing the manuscript.

Contributors RC: contributed to the experimental plan, performed research, analysed data, wrote the manuscript; CSR: contributed to the experimental plan, performed research, analysed data, commented on the manuscript; HKB, CR, PDC: performed research, analysed data, commented on the manuscript; MK, GÖL: performed research, analysed data; FB: conceived the overall research plan, analysed data, wrote the manuscript.

Funding This work was supported by the Swedish Research Council, Swedish Foundation for Strategic Research, Swedish Diabetes Foundation, Petrus and Augusta Hedlund Foundation, Torsten and Ragnar Söderbergs Foundations, Novo Nordisk Foundation, TORNADO (FP7-KBBE-222720, http://www.fp7tornado.eu/), AstraZeneca through collaboration with the Sahlgrenska Academy, and a LUA-ALF grant from Västra Götalandsregionen. CSR is the recipient of a postdoctoral fellowship from the Wenner-Gren foundation. PDC is a research associate from the FRS-FNRS (Fonds de la recherche scientifique, Belgium) and is the recipient of grants from the FSR (Fonds Spéciaux de Recherche, UCL) and the FRSM (Fonds de la Recherche Scientifique Médicale).

Competing interests None.

Provenance and peer review Not commissioned; externally peer reviewed.

\section{REFERENCES}

1. Weisberg SP, McCann D, Desai M, et al. Obesity is associated with macrophage accumulation in adipose tissue. J Clin Invest 2003;112:1796-808.

2. Xu H, Barnes GT, Yang 0, et al. Chronic inflammation in fat plays a crucial role in the development of obesity-related insulin resistance. J Clin Invest 2003;112:1821-30.

3. Lumeng CN, Bodzin JL, Saltiel AR. Obesity induces a phenotypic switch in adipose tissue macrophage polarization. J Clin Invest 2007;117:175-84.

4. Backhed F, Manchester JK, Semenkovich CF, et al. Mechanisms underlying the resistance to diet-induced obesity in germ-free mice. Proc Natl Acad Sci U S A 2007;104:979-84.

5. Rabot S, Membrez M, Bruneau A, et al. Germ-free c57bl/6j mice are resistant to high-fat-diet-induced insulin resistance and have altered cholesterol metabolism. FASEB J 2010:4948-59.
6. Ding S, Chi MM, Scull BP, et al. High-fat diet: Bacteria interactions promote intestinal inflammation which precedes and correlates with obesity and insulin resistance in mouse. PLoS One 2010;5:e12191.

7. Turnbaugh PJ, Hamady M, Yatsunenko T, et al. A core gut microbiome in obese and lean twins. Nature 2009;457:480-4.

8. Cani PD, Neyrinck AM, Fava F, et al. Selective increases of bifidobacteria in gut microflora improve high-fat-diet-induced diabetes in mice through a mechanism associated with endotoxaemia. Diabetologia 2007:50:2374-83.

9. Cani PD, Bibiloni R, Knauf $\mathrm{C}$, et al. Changes in gut microbiota control metabolic endotoxemia-induced inflammation in high-fat diet-induced obesity and diabetes in mice. Diabetes 2008;57:1470-81.

10. Membrez M, Blancher $\mathrm{F}$, Jaquet $\mathrm{M}$, et al. Gut microbiota modulation with norfloxacin and ampicillin enhances glucose tolerance in mice. FASEB J 2008;22:2416-26.

11. Cani PD, Amar J, Iglesias MA, et al. Metabolic endotoxemia initiates obesity and insulin resistance. Diabetes 2007:56:1761-72.

12. Creely SJ, McTernan PG, Kusminski CM, et al. Lipopolysaccharide activates an innate immune system response in human adipose tissue in obesity and type 2 diabetes. Am J Physiol Endocrinol Metab 2007;292:E740-7.

13. Medzhitov R. Toll-like receptors and innate immunity. Nat Rev Immunol 2001:1:135-45.

14. Reigstad CS, Lunden G0, Felin J, et al. Regulation of serum amyloid a3 (saa3) in mouse colonic epithelium and adipose tissue by the intestinal microbiota. PLoS One 2009; 4:e5842.

15. Backhed F, Soderhall M, Ekman P, et al. Induction of innate immune responses by Escherichia coli and purified lipopolysaccharide correlate with organ- and cell-specific expression of toll-like receptors within the human urinary tract. Cell Microbiol 2001:3:153-8.

16. Somerville JE Jr, Cassiano L, Bainbridge B, et al. A novel Escherichia coli lipid a mutant that produces an antiinflammatory lipopolysaccharide. J Clin Invest 1996:97:359-65.

17. Backhed F, Normark S, Schweda EK, et al. Structural requirements for TLR4mediated LPS signalling: a biological role for LPS modifications. Microbes Infect 2003;5:1057-63.

18. Karow M, Fayet 0 , Georgopoulos $\mathrm{C}$. The lethal phenotype caused by null mutations in the Escherichia coli htrB gene is suppressed by mutations in the accbc operon, encoding two subunits of acetyl coenzyme a carboxylase. J Bacteriol 1992;174:7407-18

19. Backhed F, Ding $H$, Wang $T$, et al. The gut microbiota as an environmental factor that regulates fat storage. Proc Natl Acad Sci U S A 2004;101:15718-23.

20. Everard A, Lazarevic V, Derrien M, et al. Responses of gut microbiota and glucose and lipid metabolism to prebiotics in genetic obese and diet-induced leptin-resistant mice. Diabetes 2011;60:2775-86.

21. Cinti S, Mitchell G, Barbatelli G, et al. Adipocyte death defines macrophage localization and function in adipose tissue of obese mice and humans. J Lipid Res 2005; 46:2347-55.

22. Apovian CM, Bigornia S, Mott M, et al. Adipose macrophage infiltration is associated with insulin resistance and vascular endothelial dysfunction in obese subjects. Arterioscler Thromb Vasc Biol 2008;28:1654-9.

23. Schertzer JD, Tamrakar AK, Magalhães JG, et al. Nod1 activators link innate immunity to insulin resistance. Diabetes 2011;60:2206-15.

24. Saberi M, Woods NB, de Luca C, et al. Hematopoietic cell-specific deletion of tolllike receptor 4 ameliorates hepatic and adipose tissue insulin resistance in high-fatfed mice. Cell Metab 2009;10:419-29.

25. Amar J, Chabo C, Waget A, et al. Intestinal mucosal adherence and translocation of commensal bacteria at the early onset of type 2 diabetes: molecular mechanisms and probiotic treatment. EMBO Mol Med 2011;3:559-72.

26. Hajjar AM, Ernst RK, Tsai JH, et al. Human toll-like receptor 4 recognizes hostspecific LPS modifications. Nat Immunol 2002;3:354-9. 